\title{
ON A GENERALIZATION OF THE LIZORKIN THEOREM ON FOURIER MULTIPLIERS
}

\author{
L. O. Sarybekova, T. V. TARARykova And N. T. Tleukhanova
}

Abstract. A generalization of the Lizorkin theorem on Fourier multipliers is proved. The proofs are based on using the so-called net spaces and interpolation theorems. An example is given of a Fourier multiplier which satisfies the assumptions of the generalized theorem but does not satisfy the assumptions of the Lizorkin theorem.

Mathematics subject classification (2010): 42B15, 42B35.

Keywords and phrases: Fourier multipliers, Lizorkin theorem.

\section{REFERENCES}

[1] J. Bergh, J. Lefstrem, Interpolation spaces. An Introduction, Springer-Verlag, Berlin Heidelberg New York, 1976

[2] G. H. Hardy, J. E. Littlewood, G. Polya, Inequalities, Cambridge University Press, 1934.

[3] P. I. Lizorkin, On multipliers of Fourier integrals in $L_{p, \theta}$ spaces, Trudy Matematicheskogo Instituta imeni V. A. Steklova, 1967, Vol. 139 (in Russian). English transl. in Proceeding of the Steklov institute of mathematics, 1967, Vol. 139.

[4] E. D. NURSUltanov, Nikol'skii's inequality for different metrics and properties of the sequence of norms of the Fourier sums of a function in the Lorentz space, Trudy Matematicheskogo Instituta imeni V. A. Steklova, 2006, Vol. 255, pp. 1970-215 (in Russian). English transl. in Proceeding of the Steklov institute of mathematics, 2006, Vol. 255. 